\title{
$A L D H 2$ Gene: Its Effects on the Neuropsychological Functions in Patients with Opioid Use Disorder Undergoing Methadone Maintenance Treatment
}

\author{
Po-Wei Lee ${ }^{1}$, Tzu-Yun Wang ${ }^{1}$, Yun-Hsuan Chang ${ }^{1,2,3}$, Sheng-Yu Lee ${ }^{1,4}$, Shiou-Lan Chen ${ }^{1,5,6}$, Ze-Cheng Wang ${ }^{7,8}$, \\ Po See Chen ${ }^{1,9}$, Chun-Hsien Chu ${ }^{10}$, San-Yuan Huang ${ }^{11}$, Nian-Sheng Tzeng ${ }^{11,12}$, I Hui Lee ${ }^{1,9}$, Kao Chin Chen ${ }^{1,9}$, \\ Yen Kuang Yang ${ }^{1,9,13}$, Jau-Shyong Hong ${ }^{14}$, Ru-Band Lu ${ }^{1,7,8,9}$ \\ ${ }^{1}$ Department of Psychiatry, National Cheng Kung University Hospital, College of Medicine, National Cheng Kung University, Tainan, \\ ${ }^{2}$ Department of Psychology, Asia University, ${ }^{3}$ Department of Medical Research, China Medical University Hospital, China Medical University, \\ Taichung, ${ }^{4}$ Department of Psychiatry, Kaohsiung Veterans General Hospital, ${ }^{5}$ Graduate Institute of Medicine, College of Medicine, ${ }^{6}$ Lipid Science \\ and Aging Research Center, Kaohsiung Medical University, Kaohsiung, Taiwan, ${ }^{7}$ The Affiliated Kangning Hospital of Wenzhou Medical \\ University, Wenzhou City, Zhejiang, ${ }^{8}$ Beijing YiNing Hospital, Beijing, China, ${ }^{9}$ Addiction Research Center, National Cheng Kung University, \\ ${ }^{10}$ Institute of Molecular Medicine, College of Medicine, National Cheng Kung University, Tainan, ${ }^{11}$ Department of Psychiatry, Tri-Service General \\ Hospital, National Defense Medical Center, ${ }^{12}$ Student Counseling Center, National Defense Medical Center, Taipei, ${ }^{13}$ Department of Psychiatry, \\ National Cheng Kung University Hospital, Dou-Liou Branch, Yunlin, Taiwan, ${ }^{14}$ Neurobiology Laboratory, NIH/NIEHS, Research Triangle Park, \\ NC, USA
}

\begin{abstract}
Objective: Patients with opioid use disorder (OUD) have impaired attention, inhibition control, and memory function. The aldehyde dehydrogenase $2(A L D H 2)$ gene has been associated with OUD and $A L D H 2$ gene polymorphisms may affect aldehyde metabolism and cognitive function in other substance use disorder. Therefore, we aimed to investigate whether $A L D H 2$ genotypes have significant effects on neuropsychological functions in OUD patients undergoing methadone maintenance therapy (MMT).

Methods: OUD patients undergoing MMT were investigated and followed-up for 12 weeks. ALDH2 gene polymorphisms were genotyped. Connors' Continuous Performance Test (CPT) and the Wechsler Memory Scale-Revised (WMS-R) were administered at baseline and after 12 weeks of MMT. Multivariate linear regressions and generalized estimating equations (GEEs) were used to examine the correlation between the $A L D H 2$ genotypes and performance on the CPTs and WMS-R.

Results: We enrolled 86 patients at baseline; 61 patients completed the end-of-study assessments. The GEE analysis showed that, after the 12 weeks of MMT, OUD patients with the $A L D H 2 * 1 / * 2+* 2 / * 2(A L D H 2$ inactive) genotypes had significantly higher commission error T-scores $(p=0.03)$, significantly lower hit reaction time T-scores $(p=0.04)$, and significantly lower WMS-R visual memory index scores $(p=0.03)$ than did patients with the $A L D H 21 * / * 1$ (ALDH2 active) genotype.

Conclusion: OUD patients with the $A L D H 2$ inactive genotypes performed worse in cognitive domains of attention, impulse control, and memory than did those with the $A L D H 2$ active genotype. We conclude that the $A L D H 2$ gene is important in OUD and is associated with neuropsychological performance after MMT.
\end{abstract}

KEY WORDS: Aldehyde dehydrogenase; Opioid-related disorders; Cognition; Methadone.

\section{INTRODUCTION}

Received: June 18, 2019/ Revised: October 12, 2019

Accepted: October 14, 2019

Address for correspondence: Tzu-Yun Wang

Department of Psychiatry, National Cheng Kung University

Hospital, College of Medicine, National Cheng Kung University,

138 Sheng-Li Road, Tainan 70403, Taiwan

E-mail: wangty@mail.ncku.edu.tw

ORCID: https://orcid.org/0000-0002-0561-9967
Opioid use disorder (OUD) is highly prevalent and leads to substantial social and health-related costs $[1,2]$. Compromised inhibitory control, memory, and post-error behavioral adjustment were constantly found in individuals with addiction [3]. Therefore, deficit in cogni-

(c) This is an Open-Access article distributed under the terms of the Creative Commons Attribution Non-Commercial License (http://creativecommons.org/licenses/by-nc/4.0) which permits unrestricted non-commercial use, distribution, and reproduction in any medium, provided the original work is properly cited. 
tive function might have a major role in driving OUD [4]. Studies have reported that patients with OUD had impaired cognitive and executive functions [5]. Both former and current opioid abusers had impaired cognitive flexibility, inhibition control, and working memory [6] in addition to impaired processing and psychomotor speed, verbal learning, and verbal fluency [7]. The severity of cognitive impairments was also related to the treatment outcomes in patients with OUD $[8,9]$. However, the pathophysiological mechanisms underlying these cognitive deficits associated with long-term heroin use are still unknown. Therefore, it is important to investigate the underlying etiology for cognitive dysfunction in OUD.

In addition to affecting neurocognitive functions, genetic factors might also contribute to the development of OUD [10]. We reported that the aldehyde dehydrogenase $2(A L D H 2)$ gene was associated with OUD in a Han Chinese population [11]. The $A L D H 2$ gene is on chromosome $12 \mathrm{q} 24$. A single nucleotide polymorphism $($ rs671, G $\rightarrow A$ ) in exon 12 leads to an amino acid substitution from glutamic acid (G) to lysine (A) in the ALDH2 enzyme. The ALDH enzymes have been classified as class 1 (low Km, cytosolic, including ALDH1), class 2 (low Km, mitochondrial, including ALDH2) and class 3 (high- $K m$, including ALDH3), based on kinetic properties and sequence similarities [12]. The most important enzyme for acetaldehyde oxidation are mitochondrial ALDH2, which were expressed in a larger number of tissues than ALDH1, with highest levels in liver, kidney, muscle, and heart, and also in brain [13]. ALDH2 also metabolizes dopamine and is responsible for DOPAL (3,4-dihydroxyphenylacetaldehyde) metabolism to DOPAC (3,4-dihydroxyphenylacetic acid) [14]. The G allele $\left(A L D H 2^{*} 1\right)$ encodes an active form of the ALDH2 enzyme to metabolize DOPAL, and the $\mathrm{A}$ allele $\left(A L D H 2^{*} 2\right)$ encodes an inactive form to metabolize DOPAL [15]. Other studies [16] hypothesized that the $A L D H 2$ gene is associated with OUD through dopamine metabolism, that DOPAL accumulates in opioid users with the $A L D H 2 * 2$ allele, and that this leads to opioid addiction. However, the actual mechanisms are unknown. The $A L D H 2$ polymorphisms are related to Alzheimer's disease, which implies an association between ALDH2 polymorphisms and memory function $[17,18]$. Moreover, $A L D H 2$ polymorphisms are associated with the effects of alcohol on various neurophysiological and psychomotor functions [19,20]. All these studies [17-20] hypothesized that the $A L D H 2$ gene polymorphisms affect aldehyde metabolism and cognitive function. We therefore hypothesize that the $A L D H 2$ gene affects the cognitive functions in patients with OUD as it does in alcohol abusers, and that it leads to the underlying cognitive deficits in OUD.

In the present study, we investigated the effect of $A L D H 2$ genotypes on neuropsychological performance, focus on attention, inhibition control, and memory function, all of which are central to long-term recovery in addiction [21], and in patients with OUD who have undergone methadone maintenance therapy (MMT). Because studies have reported that memory function might change after two months of MMT [22], a longitudinal study controlled for potential confounding factors is more suitable for assessing the association between changes in neuropsychological function and the $A L D H 2$ gene polymorphisms. We therefore used a longitudinal study to assess the effects of the $A L D H 2$ gene in changes of the cognitive performance of OUD patients who had undergone 12 weeks of MMT.

\section{METHODS}

\section{Participants}

The research protocol was approved by the Institutional Review Board for the Protection of Human Subjects at National Cheng Kung University Hospital (no. B-BR-103027-T). The study was done in accordance with the ethical standards laid down in the 1964 Declaration of Helsinki. The procedures were fully explained to each participant before they were asked to sign an informed consent. OUD patients were recruited from the MMT program. Each patient was initially interviewed by an attending psychiatrist and then by a research team member well trained in using the Diagnostic and Statistical Manual of Mental Disorders, fourth edition (DSM-IV) [23] criteria and the Chinese Version of the Mini International Neuropsychiatric Interview (MINI) [24]. The MINI was used because completing 4-6 hours of structured interviews, such as the Chinese Version of the Modified Schedule of Affective Disorder and Schizophrenia-Lifetime (SADS-L) [25], is difficult for OUD patients. The MINI has good reliability and has been widely used in clinical trials and epidemiological studies [26], and its interrater reliability in Chinese Version was approximately 0.75 in pre- 
vious studies $[27,28]$. Inclusion criteria were being an adult male or female between 18 and 65 years old who met the DSM-IV criteria for current opioid dependence and who used opioids daily. Exclusion criteria were having a cognitive disorder, being pregnant or nursing an infant, or having a history of one or more uncontrolled major chronic illnesses like diabetes mellitus and hypertension.

We recruited 86 OUD patients at the beginning of our study. At baseline, each patient was assessed for $A L D H 2$ genotypes and for cognitive function using the Connors' Continuous Performance Tests (CPT) and Wechsler Memory Scale-Revised (WMS-R). Their MMT and psychosocial interventions were maintained during the follow-up. After 12 weeks of follow-up, we reassessed their CPT and WMS-R.

\section{Blood Samples and Genotyping}

Twenty milliliters of venous blood was drawn from each participant, and DNA was extracted. Genotyping of the $A L D H 2$ gene was determined using protocols described elsewhere [29].

\section{Neurocognitive Test}

The Conners' Continuous Performance Test (CPT) [30] was used to assess the maintenance of focused attention and inhibitory control. An adequate level of arousal, combined with executive control to resist distraction and inhibit responses to stimuli resembling targets, were required to perform correctly. Respondents are asked to press the space bar on a computer keyboard when any letter other than " $X$ " appeared. The interstimulus intervals were 1, 2, and 4 seconds, and display time was $250 \mathrm{~ms}$. The CPT produces a standard set of performance measures that include the number of errors of omission and errors of commission. (1) Errors of omission occurred when the patient did respond to the target stimulus. (2) Errors of commission occurred when the patient responded to a nontarget $(\mathrm{X})$ stimulus. (3) Hit reaction time (HRT) represents the mean response time (ms) for all target responses over the full six trial blocks. (4) HRT standard error (HRT $\mathrm{SE}$ ) represents the consistency of response times and expresses the SE response to targets [31]. The split-half reliability is $0.66-0.95$, and the test-retest reliability after three months is $0.55-0.84$. The CPT has good reliability and validity for Han Chinese living in Taiwan [32].
The WMS-R, a major assessment of cognitive function [33], assesses all factors of memory. The WMS-R consists of 13 subtests and five indices (General Memory, Verbal Memory, Visual Memory, Attention/Concentration, and Delayed Recall). The Information and Orientation Questions (the first subtest) were used to screen for disorientation and intact sensory functioning. Attention and working memory were assessed using the Attention/ Concentration index subtests [34]. The index-score reliability coefficients range from 0.70 to 0.90 [35]. The test-retest reliability after 2 to 12 weeks is $0.62-0.82$ [33]. Interrater reliability is more than 0.9 [36].

\section{Statistical Analysis}

The differences in the frequency of the $A L D H 2^{*} 11^{*} 1$ ( $A L D H 2$ active) and $A L D H 2 * 1 / * 2+* 2 / 2(A L D H 2$ inactive) genotypes between patient and control groups were calculated using a $\chi^{2}$ test (two-tailed). Fisher's exact test was substituted for the $\chi^{2}$ test when values were smaller than expected $(<5)$. Student's $t$ test was used to estimate differences in mean age between two groups. We assigned patients with the $A L D H 2 * 1 / * 2$ and $* 2 / * 2$ genotypes to the same group, $A L D H 2$ inactive group, because the aldehyde metabolic effect as the $A L D H 2 * 2 / * 2$-encoded enzyme is inactive, and the $A L D H 2^{*} 1 / * 2$-encoded enzyme has an aldehyde metabolite rate of only $1 / 10$ that of the $A L D H 2^{*} 1 / * 1$ genotype [37]. Additionally, because there were repeated assessments, the generalized estimating equation (GEE) method [38] was used for multivariate linear regression in repeated-measures analyses that accommodate randomly missing data [39]. In the current study, GEE analysis was used to investigate the changes in CPT and WMS-R individual scores from baseline to endpoint, and in the correlations with the $A L D H 2$ genotypes. Time effects during the 12-week MMT, age, sex, methadone dose, disease duration, and psychiatric comorbidities were covariables. Significance was set at $p<0.05$. SPSS 18.0 (SPSS Inc., Chicago, IL, USA) was used for all statistical analyses.

All authors have read and approved the manuscript and declared that: (i) no support, financial or otherwise, has been received from any organization that may have an interest in the submitted work; and (ii) there are no other relationships or activities that could appear to have influenced the submitted work. 
Table 1. Demographic data and neuropsychological tests in patients with opioid use disorder

\begin{tabular}{|c|c|c|c|c|c|c|}
\hline \multirow[b]{2}{*}{ Variable } & \multicolumn{3}{|c|}{ Baseline } & \multicolumn{3}{|c|}{ Endpoint } \\
\hline & $\begin{array}{c}A L D H 2 \text { active } \\
\text { genotype }(n=40)\end{array}$ & $\begin{array}{l}A L D H 2 \text { inactive } \\
\text { genotypes }(n=46)\end{array}$ & $p$ value & $\begin{array}{c}A L D H 2 \text { active } \\
\text { genotype }(n=26)\end{array}$ & $\begin{array}{c}\text { ALDH2 inactive } \\
\text { genotypes }(\mathrm{n}=35)\end{array}$ & $p$ value \\
\hline Age (yr) & $37.4 \pm 9.2$ & $37.1 \pm 5.6$ & 0.88 & $36.5 \pm 9.2$ & $36.5 \pm 5.7$ & 0.98 \\
\hline Sex (male/female) & $33 / 7$ & $41 / 5$ & 0.38 & $24 / 2$ & $30 / 5$ & 0.42 \\
\hline Disease duration (yr) & $6.7 \pm 6.0^{\mathrm{a}}$ & $7.2 \pm 5.2^{b}$ & 0.69 & $5.7 \pm 5.9$ & $6.7 \pm 4.8$ & 0.49 \\
\hline Methadone dose (mg) & $32.13 \pm 27.78$ & $34.13 \pm 22.62$ & 0.71 & $39.81 \pm 20.37$ & $40.06 \pm 25.57$ & 0.97 \\
\hline Psychiatric comorbidities & $11(27.5)$ & $12(26.1)$ & 0.88 & $9(34.6)$ & $11(31.4)$ & 0.79 \\
\hline \multicolumn{7}{|l|}{ СРT } \\
\hline Omission T-scores & $58.91 \pm 41.68$ & $61.20 \pm 32.87$ & 0.77 & $53.68 \pm 15.56$ & $64.04 \pm 46.89$ & 0.28 \\
\hline Commission T-scores & $47.38 \pm 11.58$ & $50.50 \pm 11.38$ & 0.21 & $45.76 \pm 10.97$ & $51.28 \pm 11.81$ & 0.07 \\
\hline HRT T-scores & $52.00 \pm 14.48$ & $47.91 \pm 14.16$ & 0.19 & $54.95 \pm 14.75$ & $46.76 \pm 13.31$ & $0.03^{*}$ \\
\hline HRT SE T-scores & $53.49 \pm 13.86$ & $52.80 \pm 17.20$ & 0.84 & $58.35 \pm 12.44$ & $53.77 \pm 14.03$ & 0.19 \\
\hline \multicolumn{7}{|l|}{ WMS-R } \\
\hline Verbal Memory Index & $92.90 \pm 16.44$ & $90.07 \pm 16.49$ & 0.44 & $101.40 \pm 20.86$ & $98.18 \pm 20.14$ & 0.61 \\
\hline Visual Memory Index & $98.95 \pm 11.75$ & $94.47 \pm 14.97$ & 0.14 & $113.15 \pm 16.38$ & $99.23 \pm 14.62$ & $0.006^{*}$ \\
\hline General Memory Index & $95.23 \pm 17.56$ & $90.14 \pm 16.65$ & 0.18 & $106.15 \pm 19.74$ & $99.00 \pm 19.94$ & 0.25 \\
\hline Attention/Concentration Index & $103.43 \pm 15.47$ & $102.60 \pm 13.39$ & 0.80 & $110.50 \pm 11.82$ & $106.45 \pm 15.27$ & 0.35 \\
\hline Delayed Recall Index & $97.34 \pm 15.77^{\mathrm{c}}$ & $96.11 \pm 16.83^{c}$ & 0.75 & $106.11 \pm 18.22^{d}$ & $102.19 \pm 21.13^{\mathrm{e}}$ & 0.54 \\
\hline
\end{tabular}

Values are presented as mean \pm standard deviation, number only, or number (\%). $A L D H 2$ active genotype: $A L D H 2 * 1 /{ }^{*} 1$ genotype; $A L D H 2$ inactive genotypes: $A L D H 2 * 1 / * 2$ and $* 2 / * 2$ genotypes.

$A L D H 2$, aldehyde dehydrogenase 2; CPT, Conner's continuous performance test; HRT, hit reaction time; SE, standard error; WMS-R, wechsler memory scale-revised.

$\mathrm{a}_{\mathrm{n}}=37 ;{ }^{\mathrm{b}} \mathrm{n}=41 ; \mathrm{c} \mathrm{n}=35 ;{ }^{\mathrm{d}} \mathrm{n}=19 ; \mathrm{e}_{\mathrm{n}}=21$.

$* p<0.05$.

\section{RESULTS}

We enrolled 86 participants in our study, and 61 completed the assessments. At baseline, $46.5 \%$ of the patients had the $A L D H 2$ active genotype, and, at the endpoint, $42.6 \%$ had it. Genotype distribution of the $A L D H 2$ loci was in Hardy-Weinberg equilibrium at baseline and endpoint $(p>0.1)$. The demographic data of age, sex, disease duration, methadone dose, psychiatric comorbidities, and neurocognitive function were assessed using CPT and WMS-R. At baseline, there were no significant differences between the $A L D H 2$ active and inactive genotypes in demographic data or CPT and WMS-R scores (Table 1). However, OUD patients with the $A L D H 2$ active genotype had significantly higher HRT T-scores and WMS-R visual memory index than did patients with the $A L D H 2$ inactive genotypes at the endpoint $(p=0.03$ and $p=0.006$, respectively) (Table 1 ).

After covarying for age, sex, methadone dose, disease duration, treatment duration, and psychiatric comorbidities, the GEE analysis showed significantly different changes of commission and HRT T-scores on the CPT and WMS-R visual memory index in OUD patients with differ- ent $A L D H 2$ genotypes after 12 weeks of MMT ( $p=0.03$, 0.04, and 0.03, respectively) (Table 2).

Furthermore, OUD patients with the $A L D H 2$ inactive genotypes had significantly higher commission T- and significantly lower HRT T-scores than did those with the $A L D H 2$ active genotype during MMT (Figs. 1, 2, Table 2). OUD patients with the $A L D H 2$ inactive genotypes also had significantly lower scores on the WMS-R visual memory index than did OUD patients with the $A L D H 2$ active genotype (Fig. 3, Table 2).

\section{DISCUSSION}

This is the first study that shows the effect of the $A L D H 2$ gene polymorphisms on cognitive functions in OUD patients undergoing MMT. We found that OUD patients with the $A L D H 2$ inactive genotypes performed significantly less well in the attention and memory domain than did patients with the $A L D H 2$ active genotype. OUD patients with $A L D H 2$ inactive genotypes might react more impulsively, make more commission errors and have lower reaction times, and have poorer visual memory function during MMT than do those with the $A L D H 2$ active 
Table 2. Comparisons of the changes in neuropsychological tests after 12 weeks of MMT in groups with different $A L D H 2$ gene polymorphisms

\begin{tabular}{lrrl}
\hline & \multicolumn{3}{c}{$A L D H 2$ inactive genotypes } \\
\cline { 2 - 4 } Neuropsychological tests & B & Wald $\chi^{2}$ & $p$ value \\
\hline CPT & & & \\
Omission T-scores & 7.07 & 1.60 & 0.21 \\
Commission T-scores & 4.56 & 4.67 & $0.03^{*}$ \\
HRT T-scores & -5.18 & 4.23 & $0.04^{*}$ \\
HRT SE T-scores & -2.03 & 0.64 & 0.42 \\
WMS-R & & & \\
Verbal Memory Index & -3.29 & 1.27 & 0.26 \\
Visual Memory Index & -5.42 & 4.89 & $0.03^{*}$ \\
General Memory Index & -5.01 & 2.80 & 0.09 \\
Attention/Concentration & -0.33 & 0.02 & 0.90 \\
$\quad$ Index & & & \\
Delayed Recall Index & -2.55 & 0.54 & 0.46 \\
\hline
\end{tabular}

Reference group: $A L D H 2$ active genotype. Covarying for age, sex methadone dose, disease duration, visits, and psychiatric comorbidities.

MMT, methadone maintenance therapy; $A L D H 2$, aldehyde dehydrogenase 2; CPT, Conner's continuous performance test; HRT, hit reaction time; $\mathrm{SE}$, standard error; WMS-R, wechsler memory scalerevised.

$* p<0.05$.

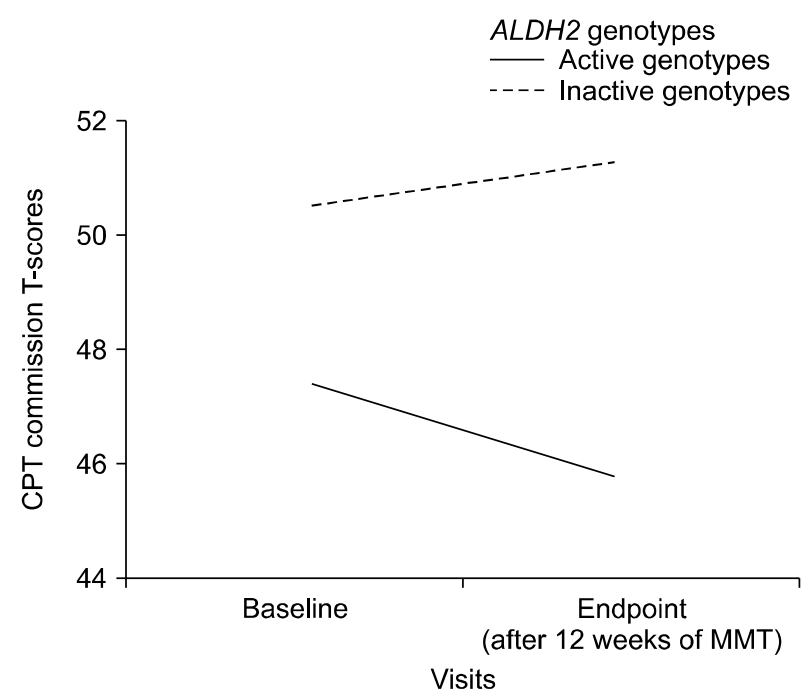

Fig. 1. Changes in CPT commission T-scores in OUD patients with different $A L D H 2$ genotypes during 12 weeks of MMT.

CPT, Conner's Continuous Performance Test; OUD, opioid use disorder; $A L D H 2$, aldehyde dehydrogenase 2; MMT, methadone maintenance therapy.

genotype. This suggests that the $A L D H 2$ gene is associated with the development and treatment outcomes in OUD patients.

Because $\mathrm{ALDH}$ is the major enzyme that metabolizes

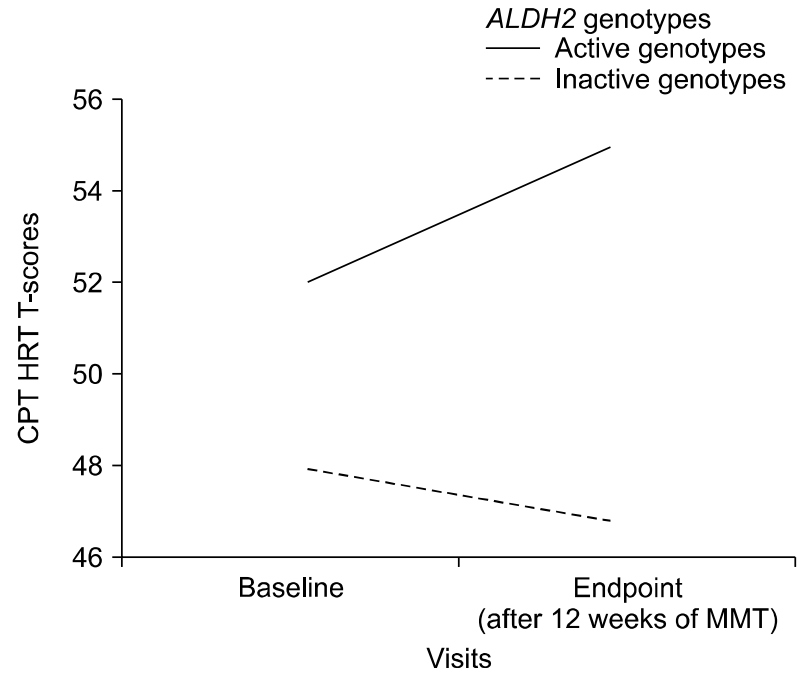

Fig. 2. Changes in CPT HRT T-scores in OUD patients with different $A L D H 2$ genotypes during 12 weeks of MMT.

CPT, Conner's continuous performance test; HRT, hit reaction time; OUD, opioid use disorder; $A L D H 2$, aldehyde dehydrogenase 2; MMT, methadone maintenance therapy.



Fig. 3. Changes in WMS-R visual memory index in OUD patients with different $A L D H 2$ genotypes during 12 weeks of MMT.

WMS-R, Wechsler memory scale-revised; OUD, opioid use disorder; $A L D H 2$, aldehyde dehydrogenase 2; MMT, methadone maintenance therapy.

acetaldehyde, it is well-known for its influence on drinking behavior and for the risk of developing alcohol dependence $[40,41]$. ALDH2 is also one of the major ALDH isozymes that catalyzes the oxidation of dopamine [37], which is an important neurotransmitter for addiction. Dopamine is metabolized by monoamine oxidase and forms DOPAL (3,4-dihydroxyphenyl-acetaldehyde), a 
toxic dopamine metabolite $[42,43]$. DOPAL is subsequently oxidized to DOPAC (3,4-dihydroxyphenylaceticacid) by ALDH $[42,44]$. Therefore, the $A L D H 2$ gene -encoded enzyme is functionally associated with the level of DOPAL $[42,44]$. Patients with $A L D H 2$ inactive genotypes might accumulate a higher level of DOPAL because the $A L D H 2$ inactive genotypes -encoded enzymes are inactive forms that metabolize DOPAL [44]. Because $\mathrm{ALDH} 2$ is widely expressed in the frontal and temporal cortex, hippocampus, midbrain, basal ganglia, cerebellum, glial cells, and neurons [45-47], the accumulation of DOPAL can consequently impair neurocognitive function, e.g., memory function. Animal studies report that ALDH2 knockdown $\left(A L D H 2^{-1-}\right)$ mice had significantly higher-than-normal hippocampal levels of toxic protein and peptides, and had age-related memory deficits and brain atrophy [17]. Human meta- analysis study also showed that $A L D H 2$ inactive genotypes increased the risk of Alzheimer's disease in East Asian men [48]. Consistent with other findings, we found that OUD patients with the $A L D H 2$ inactive genotypes had poorer visual memory function. Taking all these data together supports the notion that the $A L D H 2 * 2$ allele is associated with impaired memory function in OUD patients.

Impulsivity, associated with motor or response disinhibition, is commonly associated with addiction [49]. In addition, increased toxic aldehyde levels were found in attention-deficit hyperactivity disorder [50], which partly suggested that the deficient ALDH2 enzyme that causes aldehyde to accumulate might be associate with inattention and impulsivity. We previously reported that impulsive personality traits like novelty seeking were affected by the $A L D H 2$ genotypes in heroin-dependent patients [51]. Heroin-dependent patients with at least one $A L D H 2 * 2$ allele $(* 1 / * 2$ or $* 2 / * 2$ genotype) had significantly higher novelty seeking scores [51]. We extended the scope of other studies by objectively measuring the cognitive function associated with inhibitory controls and attention. We found that, on the CPT, OUD patients with the $A L D H 2$ inactive genotypes reacted more impulsively and had more attentional problems, more commission errors, and lower reaction times than did those with the $A L D H 2$ active genotype. Response-inhibition deficits, specifically those related to inattention and impulsive behaviors, have been related to poorer clinical treatment outcomes in addicted patients [52].
Therefore, OUD patients with the $A L D H 2$ inactive genotypes might have poorer outcomes after they undergo MMT.

Because the $\mathrm{ALDH} 2$ enzyme is required to metabolize aldehyde, it is important to highlight that ALDH2 deficiency is one of the most common enzymopathies in humans: it affects an estimated 560 million (8\%) of the world population [53]. ALDH2 deficiency is endemic in East Asia and is common in China, Japan, Korea, Taiwan, and Singapore [54]. The highest ALDH2 deficiency prevalence is in Taiwan: about half of its Han Chinese population has the $A L D H 2 * 2$ allele $[40,41]$. The ethnicity difference is important for studying the risk of developing OUD and the treatment outcomes [55,56]. Unfortunately, other studies focused only on the effects of $A L D H 2$ gene polymorphisms in modulating alcohol-related cognitive function $[19,20]$ and ignored the changes caused by other classes of substance abuse. Kim et al. [19] found that the psychomotor performance of patients with $\mathrm{ALDH} 2 * 1 / * 2$ was significantly poorer than that of those with the $A L D H 2^{*} 1 / * 1$ genotype after alcohol consumption. Our study extended the research population OUD patients and agreed with the findings of Kim et al. [19], which suggested that the $\mathrm{ALDH} 2^{*} 2$ allele was associated with substance-abuse-related neuropsychological function impairments. Additional studies to investigate whether $A L D H 2$ gene polymorphisms also affect cognitive functions in other classes of substance use disorders, such as stimulants, might be needed.

\section{Limitations}

Our study has some limitations. This is the first study that tests the hypothesis that the $A L D H 2$ gene polymorphisms are associated with neuropsychological function in OUD patients undergoing MMT. The sample size was modest; larger samples are needed. Although we controlled for some factors that might affect changes in cognitive function, other factors, e.g., the severity of comorbid psychiatric disorders, might also have affected our results. In addition, all our patients were undergoing MMT, which might have affected their attention and memory performances. Although we tried to control for the methadone dose in our analysis to reduce its effect, additional studies are required to generalize our results to abstinent former opioid abusers who did not undergo MMT. We excluded those who took other psychiatric 
medication, except methadone, in this study. However, some of the patients may still take other psychotrophic agents by themselves without informing us, which may also affect their results in neuropsychiatric tests. Therefore, our findings should be interpreted with caution.

\section{Conclusion}

OUD patients with the $A L D H 2$ inactive genotypes performed worse in cognitive domains of attention, impulse control, and memory than did those with the $A L D H 2$ active genotype. We conclude that the $A L D H 2$ gene is important in OUD and might be associated with MMT outcomes in OUD patients. Our findings must be replicated in larger samples and studies with longer follow-ups.

\section{Acknowledgments}

The study was supported by research grants from the Ministry of Science and Technology (NSC101-2314-B006-063-MY3 to RBL and MOST 106-2314-B-006-037, MOST-107-2314-B-006-067 to TYW), from the Ministry of Health and Welfare (DOH 98-TD-I-111-DD004 to RBL and MOHW107-TDU-B-211-123003, MOHW108-TD U-B-211-133003 to TYW), from the National Health Research Institutes (07A1-NPPP08-021 to RBL), from the National Cheng Kung University Project of Promoting Academic Excellence \& Developing World Class Research Centers, Taiwan (to RBL) and from the project of Integrated Drug Addiction Treatment Center, Ministry of Health and Welfare, Taiwan (to TYW).

\section{Conflicts of Interest}

All authors declare that they have no conflicts of interest.

\section{Author Contributions}

TYW and RBL designed the study and wrote the protocol. YHC, PSC, YKY and CHC supervised the laboratory work and the data analyses. TYW, SYL, KCC, SLC, NST, SYH, IHL, SHC, and RBL recruited participants. PWL wrote the first draft of the manuscript. TYW reviewed the literature and contributed to the discussion. All authors contributed to and reviewed the final version of the manuscript.

\section{ORCID}

Po-Wei Lee https://orcid.org/0000-0001-7401-3742
Tzu-Yun Wang https://orcid.org/0000-0002-0561-9967

Yun-Hsuan Chang https://orcid.org/0000-0001-8662-2457

Sheng-Yu Lee https://orcid.org/0000-0001-7796-9643

Shiou-Lan Chen https://orcid.org/0000-0003-3995-0276

Ze-Cheng Wang https://orcid.org/0000-0003-2339-8691

Po See Chen https://orcid.org/0000-0003-4963-578X

Chun-Hsien Chu https://orcid.org/0000-0003-2934-2584

San-Yuan Huang https://orcid.org/0000-0003-1268-238X

Nian-Sheng Tzeng https://orcid.org/0000-0001-5881-7089

I Hui Lee https://orcid.org/0000-0003-2970-5744

Kao Chin Chen https://orcid.org/0000-0001-8091-8820

Yen Kuang Yang https://orcid.org/0000-0001-9355-9636

Jau-Shyong Hong https://orcid.org/0000-0002-3056-8401

Ru-Band Lu https://orcid.org/0000-0001-6411-3547

\section{REFERENCES}

1. Degenhardt L, Charlson F, Mathers B, Hall WD, Flaxman AD, Johns N, et al. The global epidemiology and burden of opioid dependence: results from the global burden of disease 2010 study. Addiction 2014;109:1320-1333.

2. Grant BF, Goldstein RB, Saha TD, Chou SP, Jung J, Zhang H, et al. Epidemiology of DSM-5 alcohol use disorder: results from the national epidemiologic survey on alcohol and related conditions III. JAMA Psychiatry 2015;72:757-766.

3. Garavan $\mathrm{H}$, Hester $\mathrm{R}$. The role of cognitive control in cocaine dependence. Neuropsychol Rev 2007; 17:337-345.

4. leong HF, Yuan Z. Resting-state neuroimaging and neuropsychological findings in opioid use disorder during abstinence: a review. Front Hum Neurosci 2017;11:169.

5. Arias F, Arnsten JH, Cunningham CO, Coulehan K, Batchelder A, Brisbane $M$, et al. Neurocognitive, psychiatric, and substance use characteristics in opioid dependent adults. Addict Behav 2016;60:137-143.

6. Baldacchino A, Balfour DJ, Passetti F, Humphris G, Matthews K. Neuropsychological consequences of chronic opioid use: a quantitative review and meta-analysis. Neurosci Biobehav Rev 2012;36:2056-2068.

7. Loeber S, Nakovics H, Kniest A, Kiefer F, Mann K, Croissant B. Factors affecting cognitive function of opiate-dependent patients. Drug A/cohol Depend 2012;120:81-87.

8. Passetti F, Clark L, Mehta MA, Joyce E, King M. Neuropsychological predictors of clinical outcome in opiate addiction. Drug A/cohol Depend 2008;94:82-91.

9. Teichner G, Horner MD, Harvey RT. Neuropsychological predictors of the attainment of treatment objectives in substance abuse patients. Int J Neurosci 2001;106:253-263.

10. Tsuang MT, Lyons MJ, Eisen SA, Goldberg J, True W, Lin N, et al. Genetic influences on DSM-III-R drug abuse and dependence: a study of 3,372 twin pairs. Am J Med Genet 1996;67: 473-477.

11. Wang TY, Lee SY, Chen SL, Chen SH, Chu CH, Huang SY, et 
al. The aldehyde dehydrogenase 2 gene is associated with heroin dependence. Drug A/cohol Depend 2012;120:220-224.

12. Crabb DW, Matsumoto M, Chang D, You M. Overview of the role of alcohol dehydrogenase and aldehyde dehydrogenase and their variants in the genesis of alcohol-related pathology. Proc Nutr Soc 2004; 63:49-63.

13. Stewart MJ, Malek K, Crabb DW. Distribution of messenger $R N$ As for aldehyde dehydrogenase 1, aldehyde dehydrogenase 2, and aldehyde dehydrogenase 5 in human tissues. $J$ Investig Med 1996;44:42-46.

14. Elsworth JD, Roth RH. Dopamine synthesis, uptake, metabolism, and receptors: relevance to gene therapy of Parkinson's disease. Exp Neurol 1997; 144:4-9.

15. Huang SY, Lin WW, Ko HC, Lee JF, Wang TJ, Chou YH, et al. Possible interaction of alcohol dehydrogenase and aldehyde dehydrogenase genes with the dopamine D2 receptor gene in anxiety-depressive alcohol dependence. Alcohol Clin Exp Res 2004;28:374-384.

16. Wang TY, Lee SY, Chen SL, Chang YH, Chen $\mathrm{SH}, \mathrm{Chu} \mathrm{CH}$, et al. The ADH1B and DRD2 gene polymorphism may modify the protective effect of the ALDH2 gene against heroin dependence. Prog Neuropsychopharmacol Biol Psychiatry 2013:43:134-139.

17. D'ouza Y, Elharram A, Soon-Shiong R, Andrew RD, Bennett BM. Characterization of Aldh2 (--) mice as an age-related model of cognitive impairment and Alzheimer's disease. Mol Brain 2015;8:27.

18. Ma L, Lu ZN. Role of ADH1B rs1229984 and ALDH2 rs671 gene polymorphisms in the development of Alzheimer's disease. Genet Mol Res 2016;15:gmr.15048740.

19. Kim SW, Bae KY, Shin HY, Kim JM, Shin IS, Youn T, et al. The role of acetaldehyde in human psychomotor function: a double-blind placebo-controlled crossover study. Biol Psychiatry 2010;67:840-845.

20. Shin HY, Shin IS, Yoon JS. ALDH2 genotype-associated differences in the acute effects of alcohol on P300, psychomotor performance, and subjective response in healthy young Korean men: a double-blind placebo-controlled crossover study. Hum Psychopharmacol 2006;21:159-166.

21. Rezapour T, DeVito EE, Sofuoglu M, Ekhtiari H. Perspectives on neurocognitive rehabilitation as an adjunct treatment for addictive disorders: from cognitive improvement to relapse prevention. Prog Brain Res 2016;224:345-369.

22. Gruber SA, Tzilos GK, Silveri MM, Pollack M, Renshaw PF, Kaufman MJ, et al. Methadone maintenance improves cognitive performance after two months of treatment. Exp Clin Psychopharmacol 2006;14:157-164.

23. American Psychiatric Association. Diagnostic and statistical manual of mental. 4th ed. Washington:American Psychiatric Association; 1994.

24. Sheehan DV, Lecrubier $\mathrm{Y}$, Sheehan $\mathrm{KH}$, Amorim P, Janavs J, Weiller $\mathrm{E}$, et al. The Mini-International Neuropsychiatric Interview (M.I.N.I.): the development and validation of a structured diagnostic psychiatric interview for DSM-IV and ICD-10. J Clin Psychiatry 1998;59 Suppl 20:22-33.

25. Endicott J, Spitzer RL. A diagnostic interview: the schedule for affective disorders and schizophrenia. Arch Gen Psychiatry 1978; 35:837-844.

26. Ritchie K, Artero S, Beluche I, Ancelin ML, Mann A, Dupuy AM, et al. Prevalence of DSM-IV psychiatric disorder in the French elderly population. BrJ Psychiatry 2004; 184:147-152.

27. Kuo CJ, Tang HS, Tsay CJ, Lin SK, Hu WH, Chen CC. Prevalence of psychiatric disorders among bereaved survivors of a disastrous earthquake in Taiwan. Psychiatr Serv 2003; 54:249-251.

28. Lung FW, Cheng CT, Chang WT, Shu BC. Anxiety and mood disorder in young males with mitral valve prolapse. I Multidiscip Healthc 2008;1:89-92.

29. Dandré F, Cassaigne A, Iron A. The frequency of the mitochondrial aldehyde dehydrogenase 12 (atypical) allele in Caucasian, Oriental and African black populations determined by the restriction profile of PCR-amplified DNA. Mol Cell Probes 1995;9:189-193.

30. Conners CK, Staff M. Conners' Continuous Performance Test II (CPT II): computer program for windows, technical guide and software manual. New york:Multi-Health Systems;2000.

31. Chang YH, Chen SL, Lee SY, Chen PS, Wang TY, Lee IH, et al. Low-dose add-on memantine treatment may improve cognitive performance and self-reported health conditions in opioid-dependent patients undergoing methadone-maintenance-therapy. Sci Rep 2015;5:9708.

32. Hsieh PC, Chu CL, Yang YK, Yang YC, Yeh TL, Lee IH, et al. Norms of performance of sustained attention among a community sample: Continuous Performance Test study. Psychiatry Clin Neurosci 2005;59:170-176.

33. Wechsler D. Wechsler memory scale--revised: manual. San Antonio:The Psychological Corporation; 1987.

34. Kent P. The evolution of the Wechsler Memory Scale: a selective review. App/ Neuropsychol Adult 2013;20:277-291.

35. Elwood RW. The Wechsler Memory Scale-Revised: psychometric characteristics and clinical application. Neuropsychol Rev 1991;2:179-201.

36. O'Carroll RE, Badenoch LD. The inter-rater reliability of the Wechsler Memory Scale-Revised Visual Memory test. Br J Clin Psychol 1994;33:208-210.

37. Keung WM, Vallee BL. Daidzin: a potent, selective inhibitor of human mitochondrial aldehyde dehydrogenase. Proc Natl Acad Sci U S A 1993;90:1247-1251.

38. Zeger SL, Liang KY, Albert PS. Models for longitudinal data: a generalized estimating equation approach. Biometrics 1988; 44:1049-1060.

39. Shen CW, Chen YH. Model selection for generalized estimating equations accommodating dropout missingness. Biometrics 2012;68:1046-1054.

40. Chen CC, Lu RB, Chen YC, Wang MF, Chang YC, Li TK, et al. Interaction between the functional polymorphisms of the al- 
cohol-metabolism genes in protection against alcoholism. Am J Hum Genet 1999;65:795-807.

41. Thomasson HR, Edenberg HJ, Crabb DW, Mai XL, Jerome RE, $\mathrm{Li} \mathrm{TK}$, et al. A/cohol and aldehyde dehydrogenase genotypes and alcoholism in Chinese men. Am J Hum Genet 1991; 48:677-681.

42. Cesura AM, Pletscher A. The new generation of monoamine oxidase inhibitors. Prog Drug Res 1992;38:171-297.

43. Westerink $\mathrm{BH}$, de Vries JB. On the origin of dopamine and its metabolite in predominantly noradrenergic innervated brain areas. Brain Res 1985;330:164-166.

44. Lamensdorf I, Eisenhofer G, Harvey-White J, Nechustan A, Kirk K, Kopin IJ. 3,4-Dihydroxyphenylacetaldehyde potentiates the toxic effects of metabolic stress in PC12 cells. Brain Res 2000;868:191-201.

45. Picklo MJ, Olson SJ, Markesbery WR, Montine TJ. Expression and activities of aldo-keto oxidoreductases in Alzheimer disease. J Neuropathol Exp Neurol 2001;60:686-695.

46. Chiu CC, Yeh TH, Lai SC, Wu-Chou YH, Chen $\mathrm{CH}$, Mochly-Rosen D, et al. Neuroprotective effects of aldehyde dehydrogenase 2 activation in rotenone-induced cellular and animal models of parkinsonism. Exp Neurol 2015;263:244-253.

47. Zimatkin SM, Rout UK, Koivusalo M, Bühler R, Lindros KO. Regional distribution of low-Km mitochondrial aldehyde dehydrogenase in the rat central nervous system. Alcohol Clin Exp Res 1992;16:1162-1167.

48. Hao PP, Chen YG, Wang JL, Wang XL, Zhang Y. Meta-analysis of aldehyde dehydrogenase 2 gene polymorphism and Alzheimer's disease in East Asians. Can J Neurol Sci 2011; 38:500-506.

49. Dalley JW, Everitt BJ, Robbins TW. Impulsivity, compulsivity, and top-down cognitive control. Neuron 2014;69:680-694.

50. Bulut M, Selek S, Bez Y, Cemal Kaya M, Gunes M, Karababa $\mathrm{F}$, et al. Lipid peroxidation markers in adult attention deficit hyperactivity disorder: new findings for oxidative stress. Psychiatry Res 2013;209:638-642.

51. Lee SY, Wang TY, Chen SL, Huang SY, Tzeng NS, Chang YH, et al. Interaction between novelty seeking and the aldehyde dehydrogenase 2 gene in heroin-dependent patients. J Clin Psychopharmacol 2013;33:386-390.

52. Albein-Urios N, Pilatti A, Lozano O, Martínez-González JM Verdejo-García A. The value of impulsivity to define subgroups of addicted individuals differing in personality dysfunction, craving, psychosocial adjustment, and wellbeing: a latent class analysis. Arch Clin Neuropsychol 2014;29:38-46.

53. Brooks PJ, Enoch MA, Goldman D, Li TK, Yokoyama A. The alcohol flushing response: an unrecognized risk factor for esophageal cancer from alcohol consumption. PLoS Med 2009;6:e50

54. Chen $\mathrm{CH}$, Joshi AU, Mochly-Rosen D. The role of mitochondrial aldehyde dehydrogenase $2(A L D H 2)$ in neuropathology and neurodegeneration. Acta Neurol Taiwan 2016;25:111. 123.

55. Che Y, Assanangkornchai S, McNeil E, Chongsuvivatwong V, Li J, Geater A, et al. Predictors of early dropout in methadone maintenance treatment program in Yunnan province, China. Drug A/cohol Rev 2010;29:263-270.

56. Hser YI, Evans E, Huang D, Weiss R, Saxon A, Carroll KM, et al. Long-term outcomes after randomization to buprenorphine/naloxone versus methadone in a multi-site trial. Addiction 2016;111:695-705. 